\title{
A Process Model for RFID based Business Process Analysis
}

\author{
Thomas Neubauer, Gernot Goluch, Stefan Jakoubi, Simon Tjoa, Martin Wisser \\ Secure Business Austria \\ Vienna, Austria \\ \{neubauer, goluch, jakoubi, tjoa, wisser\}@securityresearch.ac.at
}

\begin{abstract}
In an agile and dynamic market, companies face the challenge to continually adapt their business processes. Technologies such as RFID allow companies to automatically monitor their business processes. This paper introduces RFID based Business Process Analysis (RBPA). With the usage of RFID transponders and readers, different types of data about existing business processes is acquired and used as input for a detailed process analysis and simulation as well as for the automatic generation of process models. The approach supports process managers in executing detailed business process analysis with less effort and more adequate parameters compared to existing approaches.
\end{abstract}

Keywords- RFID; Business Process Analysis

\section{INTRODUCTION}

Today enterprises face a very dynamic and agile market [4]. In order to survive those fast changing circumstances and stay competitive, companies continually have to adapt their business processes. Additionally, most CEOs believe that their companies are not adaptive and agile enough to cater to changes in their current markets and at the same time pursue emerging opportunities. As this study shows, CEOs (i) urgently want to implement a competitive intelligence capability that allows the organization to react quickly and (ii) must anticipate and respond almost intuitively to changing customer needs [14]. With the use of information technology, business processes changed radically in the last four decades and the concept of enterprise has evolved dramatically as its functionalities and boundaries have extended in response to business and technological changes [2]. Yet, until this day, the integration between IT and the real world is a main problem [6]. The discontinuity between the abstract virtual representation and the real world is still costly.

By using RFID [12] technology and methodologies, companies can automatically adjust their processes to a changing environment and minimize the gap among virtual and real world. Today it is standard to use random sampling to track and trace items through a workflow process. To trace and identify more items, bar codes are used although they have a lot of disadvantages in the recognition process [11]. Due to RFID's ubiquities characteristics it is possible to track all items through different workflows or even through their whole lifecycle, to measure the time they rest or the time they move from one station to another. In combination with sensors, data about the environment like temperature of humidity could be recorded and used in detailed process analysis. AMR Research [17] asked nearly 500 companies about their plans related to RFID technology. A third of them were evaluating the use of RFID until 2006, whereas a quarter of the questioned had running pilot projects. The range of applications varies from Track and Trace, inventory management, asset tracking to quality control. Following this, RFID technology can be seen as a major opportunity for companies to change, and adapt respectively, their business processes. The return on investment depends on how they make use of RFID's capabilities. For example, if companies only use RFID as a bar code replacement and don't change their underlying business process, the result will be suboptimal. To stay competitive, processes have to be adapted and optimized for the use of RFID. Therefore it is necessary to understand the existing process [1] and to analyze the workflow of daily operations. About $60 \%$ of time, business process management focuses on the acquisition and analysis of the existing processes [9]. Decreasing this number by the appliance of new technologies and corresponding new approaches will on the one hand reduce costs and save resources regarding business process management activities, and on the other hand increase the quality of extracted data. Giving precise instructions, the output of such an analysis leads to shorter periods and improves process quality and transparency (cf. [1]). Therefore detailed workflow analysis can be done in less time with more measured factors. Today the main application of RFID is in supply chain event management (more than 60\%). Different application fields, such as RFID based process analysis, will become more attractive, due to two reasons: (i) The price of RFID transponders, detectors and related middleware have fallen to a level, where more and more applications become reasonable; (ii) The upcoming RFID technology forces companies to make use of the new opportunities in order to stay competitive. This paper presents - based on a roadmap paper (cf. [14]) - a process model for RFID based business process analysis (RBPA) consisting of four phases: (1) Initialization including the preparation of business process models and the definition of main goals. (2) Conceptual design including the selection and evaluation of proper technology. (3) Execution of the business process analysis. (4) Evaluation and utilization of the acquired data. This new approach provides process managers more detailed data from business process analysis and supports the automatic generation of process models with less effort and more adequate parameters compared to existing approaches. 


\section{BACKGROUND}

To analyze a business process using computer systems, automatic identification is the most important requirement. Only complete registration of all relevant data enables the system to map the real world data to the virtual representation. Depending on the application, manual or automatic data acquisition is conceivable. Three techniques are widely used for automatic identification: (1) Optical character recognition, (2) bar codes and (3) RFID. The main applications of RFID in this area are automatic labeling, identification, registration, control and transport.

In the past, RFID was used for the identification of animals, cars and access control. Since companies like Wal-Mart and institutions like the US Department of Defense (cf. [13]) use RFID in their logistic chain the importance of RFID for business process optimization is continuously growing. Despite this trend the integration between IT and the real world remains the main research problem (see Fig. 1). The Supply Chain Council, which is supported by academic and industrial members developed to SCOR model to illustrate and identify supply chain processes. It contains a standard description of management processes, describes the relationships between the processes and offers standard metrics to measure process performance [10]. We choose this reference model because it offers not only a process description but also aids with process identification. SCOR is not tied to any specific industry and is used to illustrate, analyze and configure supply chains from the supplier's supplier to the customer's customer. The following itemization outlines the scope of the five SCOR core processes [17]:

- Plan: Demand and supply planning and management;
This process contains all procedures to balance resources with requirements and communicates plans for the supply chain. Additionally it tries to align the supply chain unit plan with the financial plan.

- $\quad$ Source: This process describes all activities needed to assure the companies provisioning with raw material, trade goods and services and it identifies and selects the supply sources.

- Make: Schedules all production activities including produce and test, package and release product to deliver. According to Huang et al. "Make" is the core process of the system in which the production execution takes place [10])

- Deliver: The core procedures are order management, transportation and installation management. In addition this process describes the warehouse management from receiving material to shipping the product.

- Return activities: This process describes the receiving and handling of return products.

Furthermore the SCOR model consists of three levels which offer different process details: Level one is the "Top Level" where the scope and the content for the supply chain reference model is defined. The top level deals with process types. The "Configuration Level" is the second level and deals with process categories. Additionally, the operations strategy of a company is implemented in this level. The "Process Element Level" is the lowest level specified in SCOR. It defines company's ability to compete in its chosen markets [10]).

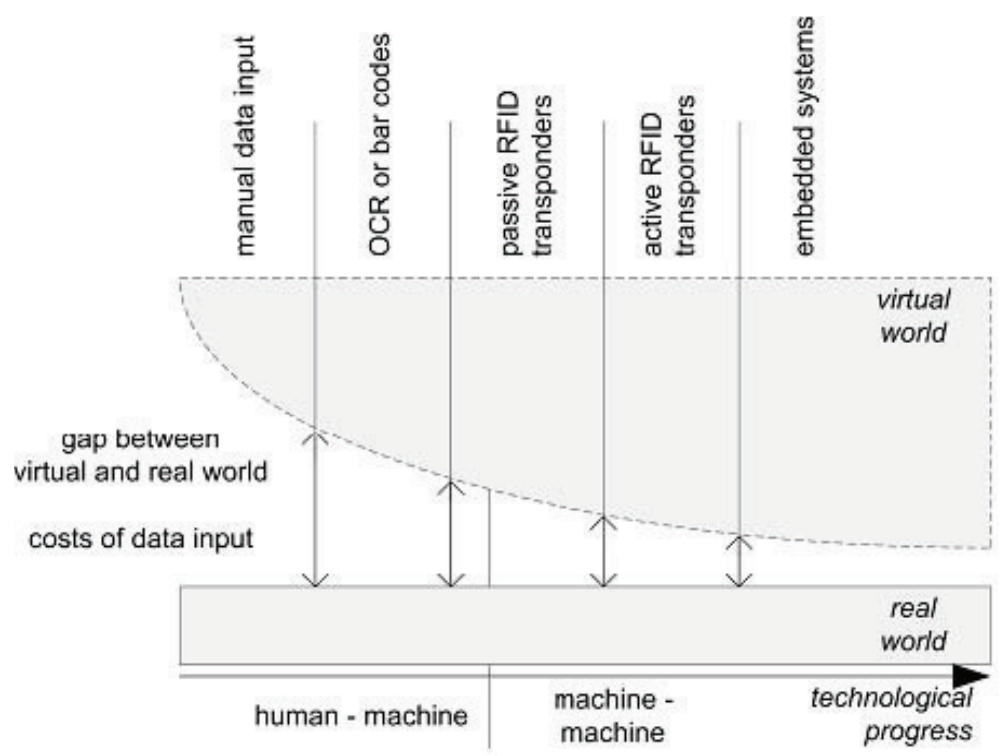

Figure 1. Integration of virtual and real world [5, 17] 


\section{RFID BASED BUSINESS PROCESS ANALYSIS}

Our RFID Based Business Process Analysis (RBPA) process model contains four phases (see Fig. 2): (1) Initialization: Preparation of business process models and the definition of main goals. (2) Conceptual design: Selection and evaluation of proper technology. (3) Execution: Execution of the business process analysis. (4) Evaluation: Evaluation and utilization of the acquired data.

\section{A. Initialization}

The main goal of the initialization phase is the assurance of proper business process descriptions and models as a starting point for process analysis. Therefore a systematic approach is needed, considering the persons, objects, business processes, information systems, environmental conditions and strategic goals of the company (cf. [7]). Time and effort of this phase may differ from company to company due to the existence of and prioritized. Key aspects regarding a business process, which can be answered by RBPA, are process value, running time, process costs, process quality, organizational aspects, system inconsistencies and efficiency. The running time can be analyzed for one specific order type or one production compartment. The tracking of vehicles on the premises is not related to specific production compartments but to the whole site. The coverage depends on the objectives and the company's strategic goals, but directly affects the design phase and analysis costs. The next step uses the objectives and coverage as input for the identification of business processes relevant to the analysis. The focus and the level of detail should depend on both, objectives and coverage. Depending on the company there might already be a proper business process description, which can be used as a starting point for RBPA. Valuable starting points for this step are process inputs and outputs, costs, revenue or value. Each process transforms some input into some output, which creates expenditures. Following

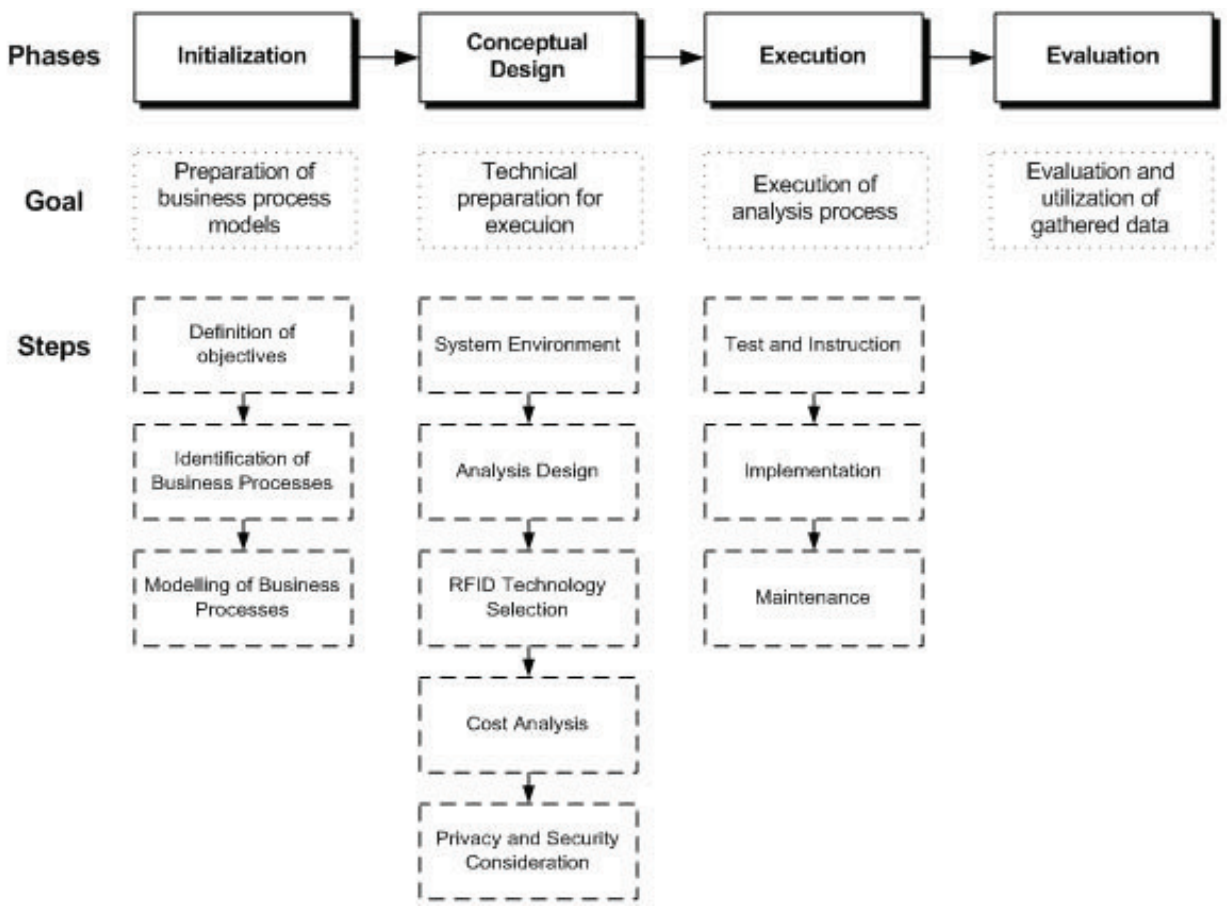

Figure 2. Phases, goals and steps of RBPA

proper process documentation. This phase consists of three steps (see Fig. 2):

- Definition of Objectives

- Identification of Business Processes

- Modeling of Business Processes

The aim of Business Process Analysis (BPA) is to offer new information about existing business processes to gain a general understanding of the process and the environment [1]. In the first step the objectives and the coverage of the analysis are defined. Therefore the processes main goals are identified this, the identification of costs as well as of generated values can lead to business processes. According to [4], business processes can be divided recursively into sub processes, activities and tasks. Before proceeding to the next step, the level of detail for the processes respectively the sub processes has to be defined. For RBPA as for any business process analysis, a certain level of detail of the process description is needed (cf. $[4,1])$. To be able to acquire exact data about the supply chain and the connected processes, the description should reach to the task level. For example, if the goal is to identify the running time of an order, all the elements have to be considered (e.g. total process time, idle time, transfer time). These different elements are evaluated and simulated in the 
third and fourth RBPA phase, if the process description allows the assignment of the gathered data to the process model. Two types of analysis approaches are conceivable, the first one starting with a detailed process model and the second one leading to a detailed process model. The difference is the level of granularity provided by the process model in this stage of RBPA. Either the process model offers details up the task descriptions and will be added with additional information about the process or the process model is more general and the RBPA is used to acquire additional information about the process to complete the process model in the first step and to analyze and simulate the process in the second step $[15,16]$. In both cases, a process model with a certain level of detail is needed as a starting point. Different modeling languages are commonly used to describe and model business processes: Object Property Relationship (OPR), Event-driven Process Chain (EPC), Unified Modeling Language (UML), Petri Nets, Integrated Definition Method 3 (IDEF3), Business Process Modeling Notation (BPMN) and Agent Relationship Morphism Analysis (ARMA) ([17, 4, 18]). Business process modeling methodologies can be characterized into general modeling methodologies and methodologies for a specific application like workflow management or quality management [1]). At this point it is not important which modeling technique is used to describe the business process. In some cases, the company's ERP system restricts the choice, like ARIS favorites EPCs. On the other hand, many simulation approaches or tools use Place/Transition Nets to illustrate their process model and to offer a look into the future with simulating the process behavior under specific circumstances [16]. So the selection of the proper modeling language used to describe the business process models depends on several aspects. The choice should be made under consideration of the predefined objectives and the coverage of the analysis.

\section{B. Conceptual Design}

Constitutive on the analysis's objectives and coverage, the target business processes, their process model, the system environment and the premises layout are analyzed. With this information, the proper RFID technology can be selected and costs can be estimated. This phase consists of five steps (see Fig. 2):

- System Environment

- Analysis Design

- RFID Technology Selection

\section{- Cost Analysis}

Many companies support their business processes with Process Aware Information Systems (PAIS) [15]. The aim of this step is to connect these systems to a RFID system. Therefore, RFID suppliers offer edgeware to connect the RFID hardware to an existing system (e.g., SAP). The main advantage of such middle- and edgeware is that the RFID hardware can be chosen independently from the existing system. That is important for the next step of our process model, the RFID technology selection. The selection should be made under consideration of feasibility issues. Often the proof of feasibility can only be made in close collaboration with
RFID technology vendors [17]. Before the specific RFID technology is selected, an analysis design is needed. There are three questions which have to be answered in this step: What will be measured? Where will it be measured? How will it be measured? The answers are strongly bound to the selection of the specific RFID technology, but should be kept at a higher level. At this stage it is not important, for example, which type of gate reader from which vendor is used, but that some gate reader is used at a specific place to read data from transponders on incoming products. The objectives defined in the previously explained first RBPA phase represent the base for the answer of the first question. With Auto-ID technology, like bar codes or RFID, different types of data can be gathered about a business process. One possibility is the recording of timestamps when the transponders pass through the gate readers. Another option is to record information with mobile readers, handled by employees. RFID vendors offer different solutions to record location data of transponders, so the routing of products or containers can be registered. Up to date RFID transponders offer various options for recording environment data. Therefore, they can be combined with different types of sensors [1]. After defining what data is going to be measured, the next question to be answered is where this data can be measured with minimal effort. To record time data about a task, the reader has to be placed spacial in front and after the task's place. The tagged containers or products are recorded when they arrive at the workplace and when they leave the workplace. Another possibility is to readout data manually before the task starts and when it ends. Depending on the type of localization system, the location of the readers is given by system requirements to achieve a sufficient coverage. Key factors, which are important to select the proper reader type, are reflections, reader size and environmental conditions. The fourth step of the design phase is the selection of proper RFID technology. This step strongly influences the measurement result of the analysis. For an adequate selection, not only organizational information about the processes is needed, but also information about related resources and equipment. The desired data about the business processes or the task is already defined, as well as the location for the readers and the reader types. At this step, the precise selection of the transponder and reader technology is made. Depending on the requirements for the analysis and the surrounding conditions, the first choice to make is the proper frequency. The transponders' and readers' characteristics depend on the frequency used to transfer the data signal. The range varies from low frequency (LF) with $100-135 \mathrm{kHz}$ to ultra high frequency (UHF) with $868 \mathrm{MHz}$ or $915 \mathrm{MHz}$. With rising frequency, the antenna size decreases, the attenuation by water declines as well as the energy demand. On the other hand, reflection and orientation effects of the antenna are increasing. One important advantage of higher frequencies is the higher data rate. In addition, most reader types are only available for high frequency (HF) or UHF transponders. The frequency also affects the signal distance, thus the maximal distance between the transponder and the reader. For most of the given data examples, vicinity systems with a reader distance up to one meter are sufficient. A longer reading distance increases the possibility for miss measurement when transponders come into the reading distance of a reader unintended. For exact location data, long-range systems are 
needed, e.g., for triangulation algorithms. Depending on the process, different multi-tag coordination techniques are applicable. If many transponders have to be readout by the reader, eventually with sensor and GPS data, the selection of readers with the proper multi-tag coordination technique is critical for a meaningful analysis [8]. An additional factor for the selection of proper RFID hardware is parasitic frequencies. Those frequencies can result in miss measurements and can be caused by wireless or cell phone systems, if they run at the same frequency range as the selected RFID transponders and readers [17]. Another important factor is that the natural sequence of work steps should not be affected by the RFID data acquisition. Significant analysis data can only be measured if the underlying business process runs unchanged. Passive, active and semi-active transponders are available in different formats. The decision, which transponder type fits best for the demanded requirements is foreclosed by the other design factors. For long-range system or systems with sensors or GPS bounded to the transponder, only active powered transponders will suit. Security and privacy issues are a main point for all RFID based applications. Security concerns should be considered in the design phase, especially when the specific hardware is selected, since the possible attacks are different depending on the applied RFID hardware. But not only the RFID hardware and the communication between reader and transponder should be secured, also the data transmission from the reader to the IT system. Wired communication might be more expensive and in some cases not reasonably applicable, but wireless communication offers some security risk, which should be considered [3].

\section{Execution}

In this phase, the actual data acquisition takes place. Execution contains test of technology at the premises, instructions and support for staff members, the installation of the RFID transponders and readers and their connection to the IT system. The steps of this phase are (see Fig. 2):

- $\quad$ Test and Instruction

- Implementation

\section{- Maintenance}

In the first step design parts have to be tested at their place of installation. To be sure that all assumptions made in the design process are correct, testing of the specific RFID devices is needed. Possible test cases are the signal distance between reader and transponder, possible reflection and deflection by material near to the place of installation or the communication with the existing system. Additionally the sensors or GPS modules should be tested and in case of other location techniques, the resolution and coverage should be investigated. Additionally the instruction of staff members dealing with the RFID system (hardware and middle- or edgeware) has to be performed, providing an overview about what is being done and how the changes affect their workplace. After the initialization of the transponders they can be attached to the products, containers, tools or other devices depending on the analysis objectives and design. The readers have to be placed at their designated point of installation and connected to power supply and the IT system. The RFID middle- or edgeware has to be integrated into the IT system and tested as well as all the other devices. During the utilization of the RFID system, regular inspections of all devices are needed.

\section{Evaluation}

The Evaluation phase can be run parallel to the, depending on the objectives and the design of the analysis. The aim is to answer the questions defined in the first phase of the analysis and to accomplish the defined objectives.

\section{AREAS OF APPLICATION}

Two possible areas for the application of RBPA are conceivable: (1) RFID as an advanced Auto-ID technology for a temporary analysis of existing business processes and (2) RFID in combination with data mining tools used for a longer period of time for business process improvement. Regarding the first option the process model is developed or updated in the initialization phase before the conceptual design takes place. The data acquired by RFID devices is later used to add additional information to the process model, e.g., for simulation or cost analysis of the process. The modeling language may differ from company to company, since the language has to be supported by the existing IT system. A possible choice is the ARMA methodology, which supports all three perspectives of business process redesign: Management accounting, information system development and organizational theoretic perspective [18]. ARMA offers a wide spectrum of analysis approaches with different objectives. Some of them are placed on an organizational level like the analysis of abstraction levels or the management structure analysis, while others like process cycle time analysis concentrate on optimizing cross-functional business processes. The process perspective is illustrated with object lifecycles (OLCs) an offer a great possibility to add business process data to the model [18]. Focusing on the second application scenario, a combination of the described RBPA approach with data mining tools for automatic generation of business process models is used. In most approaches petri nets are chosen to describe the business process models and to offer the possibility for simulation. Data mining techniques can be used for process discovery and conformance checking based on data from real process executions [15]. Process discovery describes the procedure to readout event logs from observed real life processes and construct a process model based on that information. With conformance checking, deviation between real life processes and the recorded behavior can be detected $[19,15]$. These process models need up to date data about the processes. ERP systems like SAP usually log information about who is doing what in combination with timestamps [19]. If the data range could be expanded, for example, with the use of RFID devices, the analysis approach would move towards Autonomous Business Process Analysis and ABPM (cf. [14]).

\section{CONCLUSIONS}

In this paper, we presented how RFID, as an Auto-ID technology, can be used for business process analysis. The identified area of application of RFID in business process management is currently supply chain management [17]. Thereby, the new approach provides improved data quality and 
real time data about the business process. Today, research and practical experience is concentrated on connecting objects, people and processes. With RFID, the gap between the real world and the abstract, virtual representation is reduced. For analyzing a process, active transponders in combination with sensors or GPS modules offer the most interesting possibilities. To readout the information of the transponders, different types of reader can be used, depending on the actual environment and analysis objectives. The main potential of RFID for the automatic data acquisition regarding business processes lies in: (1) automatic identification without a direct line of sight, (2) possibility to combine identification with information about the environment and (3) possibility to track and trace single objects The SCOR model was used to identify supply chain processes, which can be analyzed based on RFID technology. The data acquisition starts with receiving and verifying the products and ends with the delivery. The acquired data ranges from time data of process activities, hold-ups to sensor data like temperature, acceleration and location data. Further work will focus on conducting case studies with our partner companies in order to provide empirical evidence about the effectiveness of this approach in practice.

\section{ACKNOWLEDGMENT}

This work was performed at the research center Secure Business Austria funded by the Federal Ministry of Economy, Family and Youth of the Republic of Austria and the City of Vienna.

\section{REFERENCES}

[1] T. Allweyer. Geschäftsprozessmanagement, Strategie, Entwurf, Implementierung, Controlling. W31, 2005.

[2] M. Castellanos, F. Casati, M. Sayal, and U. Dayal. Challenges in business process analysis and optimization. In Lecture Notes in Computer Science, volume 3811, pages 1-10, 2006.

[3] H. Chan and A. Perrig. Security and privacy in sensor networks. Computer, 36(10):103-105, 2003.
[4] G. Darnton and M. Darnton. Business Process Analysis. Thomson Business Press, 1997.

[5] E. Fleisch and F. Mattern. Das Internet der Dinge, chapter Die betriebswirtschaftliche Vision des Internets der Dinge, pages 3-38. 2005 .

[6] F. Gillert and W.-R. Hansen. RFID für die Optimierung von Geschäftsprozessen. . Carl Hanser Verlag Mnchen Wien, 2007.

[7] S. Gross and F. Thiesse. Das Internet der Dinge, chapter RFID-SystemEinführung-Ein Leitfaden für Projektleiter, pages 303-314. 2005.

[8] T. Hassan and S. Chatterjee. A taxonomy for RFID. In Proceedings of the 39th Hawaii International Conference on System Sciences, 2006.

[9] J. Herbst and D. Karagiannis. An inductive approach to the acquisition and adaptation of workflow models. In Proceedings of the 16th International Joint Conference on Artificial Intelligence (IJCAI '99), 1999.

[10] S. Huang, X. Wang, and A. Zhou. Efficient web service composition based on syntactical matching. pages 782-783. IEEE Computer Society, 2005

[11] H. Kim, A. Sengupta, and J. Evermann. MOQ: Web services ontologies for QoS and general quality evaluations. In European Conference on Information Systems (ECIS), 2005.

[12] M. Lampe, C. Flörkemeier, and S. Haller. Das Internet der DingeUbiquitous Computing und RFID in der Praxis, chapter Einführung in die RFID-Technologie, pages 69-86. Springer-Verlag, 2005.

[13] M. McGinity. RFID: is this game of tag fair play? Commun. ACM, 47(1):15-18, 2004.

[14] T. Neubauer and C. Stummer. Extending business process management to determine efficient IT investments. In Proceedings of the 2007 ACM symposium on Applied computing, pages 1250-1256, 2007.

[15] A. Rozinat and W. v. d. Aalst. Decision mining in business processes. In BETA Working Paper Series (Int. rep. WP 164). Onderzoekschool Beta., 2006.

[16] A. Rozinat, R. Mans, M. Song, and W. v. d. Aalst. Discovering simulation models. In Beta working paper series (Int. rep. WP 223). Onderzoekschool Beta., 2007.

[17] D. Schmidt. RFID im Mobile Supply Chain Management Betriebswirtschaftlicher Verlag Dr. Th. Gabler, Wiesbaden, 2006.

[18] G. Valiris and M. Glykas. Business analysis metrics for business process redesign. Business Process Management Journal, 10(4):445-448, 2004

[19] W. M. P. van der Aalst. Patterns for Process-Aware Information Systems. BPM Center Report, pages 1-13, 2004. 